\title{
TMSmap - Software for Quantitative Analysis of TMS Mapping Results
}

\author{
Pavel A. Novikov ${ }^{1 \dagger}$, Maria A. Nazarova ${ }^{1 \dagger}$ and Vadim V. Nikulin ${ }^{1,2,3 *}$ \\ ${ }^{1}$ Centre for Cognition and Decision Making, Institute for Cognitive Neuroscience, National Research University Higher School \\ of Economics, Moscow, Russia, ${ }^{2}$ Department of Neurology, Max Planck Institute for Human Cognitive and Brain Sciences, \\ Leipzig, Germany, ${ }^{3}$ Neurophysics Group, Department of Neurology, Campus Benjamin Franklin, Charité-Universitätsmedizin \\ Berlin, Berlin, Germany
}

\section{OPEN ACCESS}

Edited by:

Hans-Leo Teulings,

Neuroscript, United States

Reviewed by:

George Wittenberg,

University of Maryland School of

Medicine, United States

Emilia Ambrosini

Politecnico di Milano, Italy

${ }^{*}$ Correspondence:

Vadim V. Nikulin

nikulin@cbs.mpg.de

tThese authors have contributed equally to this work and share the first authorship.

Received: 06 December 2017 Accepted: 24 May 2018 Published: 09 July 2018

Citation:

Novikov PA, Nazarova MA and Nikulin W (2018) TMSmap - Software for Quantitative Analysis of TMS Mapping Results.

Front. Hum. Neurosci. 12:239.

doi: 10.3389/fnhum.2018.00239
The use of the MRI-navigation system ensures accurate targeting of TMS. This, in turn, results in TMS motor mapping becoming a routinely used procedure in neuroscience and neurosurgery. However, currently, there is no standardized methodology for assessment of TMS motor-mapping results. Therefore, we developed TMSmap-free standalone graphical interface software for the quantitative analysis of the TMS motor mapping results (http://tmsmap.ru/). In addition to the estimation of standard parameters (such as the size of cortical muscle representation and the center of gravity location), it allows estimation of the volume of cortical representations, excitability profile of the cortical surface map, and the overlap between cortical representations. The input data for the software includes the coordinates of the coil position (or electric field maximum) and the corresponding response in each stimulation point. TMSmap has been developed for versatile assessment and comparison of TMS maps relating to different experimental interventions including, but not limited to longitudinal, pharmacological and clinical studies (e.g., stroke recovery). To illustrate the use of TMSmap we provide examples of the actual TMS motor-mapping analysis of two healthy subjects and one chronic stroke patient.

Keywords: motor cortex, transcranial magnetic stimulation (TMS), cortical mapping, earth mover's distance (EMD), overlap, interpolation on the sphere, free software

\section{INTRODUCTION}

Transcranial magnetic stimulation (TMS) is a non-invasive approach for studying brain organization in humans. In TMS time-varying magnetic fields generate electrical currents in the targeted brain regions resulting in the activation of the neuronal tissue (Ruohonen and Karhu, 2010). The magnetic field strongly attenuates with increasing distance from the coil and, therefore, the brain areas stimulated with TMS are located rather superficially at a distance of about $2-4 \mathrm{~cm}$ under the skin (Groppa et al., 2012), which corresponds roughly to the gray-white matter border. In order to identify the stimulated cortical area, a precise location of the coil should be determined with respect to the individual's brain anatomy. This can be achieved with a combination of magnetic resonance imaging (MRI) data, and devices tracking the position of the subject's head and TMS coil, so-called navigated TMS (nTMS) approach which provides a navigation with millimeter accuracy (Krieg, 2017). Such spatial accuracy is particularly useful for TMS cortical mapping where the brain is stimulated at small and usually regularly spaced points. Theoretically, any response to TMS elicited from a specific cortical point, can be used for TMS mapping. In principle, TMS mapping can be specified with the following two parameters: (1) type of TMS protocol: for instance, single pulse, or paired pulse TMS-routinely used for motor TMS mapping, repetitive TMS - used for 
speech areas and so on; (2) type of the measured biological activity: electromyography (EMG) for motor cortex mapping, behavioral responses (phosphenes, reaction times, hit-rate, errors, etc.), electroencephalography (EEG), functional MRI (fMRI) etc. Such a variety of protocols and neurophysiological measures indicates that TMS mapping represents a promising approach for the non-invasive investigation of the brain in different fields of neuroscience.

Presently, however, only presurgical motor and speech nTMS mapping has received widespread use (Krieg et al., 2017). Yet the use of TMS mapping for the investigation of cortical neuroplastic changes, after surgery, during rehabilitation, training, or for any other longitudinal investigations, is still limited both in clinical and basic research environments. This is despite the fact that TMS compared, for example, to functional MRI, reflects the link between the structure and function in a direct causal way and usually requires less compliance from a subject. There are several reasons for such situation. Firstly, even for TMS motor mapping, there is no general agreement about the exact parameters describing cortical representations. Moreover, even most commonly used parameters such as areas, volumes, the location of centers of gravity (CoGs), and hotspots have not been validated for being reliable measures in test-retest studies (Kraus and Gharabaghi, 2016). Eventually, even for the extensively studied field of stroke recovery the lack of the standardized methodology leads to considerable difficulties when summarizing the findings of the publications with TMS motor mapping (Lüdemann-Podubecká and Nowak, 2016). Secondly, despite the long history of TMS motor mapping, one should still develop a standardized toolbox or software for the quantitative analysis of TMS mapping results. Yet, a unified workflow would alleviate results' comparisons across sessions, subjects and studies.

In order to address these challenges, we present TMSmapfree standalone graphical interface software for quantitative analysis of the TMS mapping results (http://tmsmap.ru/, registration number RU 2016614899, 11.05.2016, a freeware license). It provides an integrative approach for the complex assessment and visualization of TMS motor mapping results. The software includes assessment of the standard features of TMS maps, such as areas and volumes of the cortical representations, CoGs, and hotspots, as well as novel parameters such as cortical representations' excitability profiles and the overlaps between the cortical representations-showing, for example, coactivation of the different muscles.

The purpose of this article is to describe the software options and workflow, primarily, for the quantification of TMS motor mapping data. To illustrate the programs' capabilities, we use examples of nTMS multi-muscle motor mapping in two healthy

\footnotetext{
Abbreviations: ABOS, approximation based on smoothing; ADM, abductor digiti minimi; $\mathrm{AH}$, affected hemisphere; $\mathrm{APB}$, abductor pollicis brevis; CS, coordinate system; CoG, center of gravity; EDC, extensor digitorum communis; EEG, electroencephalography; EF, electric field; EMD, earth mover's distance; EMG, electromyography; fMRI, functional magnetic resonance imaging; MEP, motor evoked potential; MRI, magnetic resonance imaging; nTMS, navigated transcranial magnetic stimulation; POI, patch of interest; r_map, raw map; RMT, resting motor threshold; TMS, transcranial magnetic stimulation; th_map, thresholded map; UH, unaffected hemisphere.
}

subjects and in one chronic stroke patient with hand motor deficit.

\section{METHOD-SOFTWARE}

TMSmap software is standalone software with a graphical interface (Figure 1). It was written using WPF (C\# and XAML), based on .NET Framework 4.5.2, and it supports Microsoft Windows operating system version 7 or higher. TMSmap is a freeware: one can download and use it free of charge. In this section, we present TMSmap possibilities and general data workflow. We will also briefly describe methods and approaches for data analysis and visualization. As this article is not intended to be a user manual, we invite the reader to visit the website http:// tmsmap.ru/ for further detailed information including video demonstrations.

TMSmap allows a fast construction and visualization of the 3D maps of cortical representations and a calculation of the parameters that are most commonly used in TMS mapping analysis such as: areas of representation, centers of gravity (CoG) locations, hotspots locations, and volumes of representation. In addition, we introduce several new mapping approaches: (1) a quantitative comparison of the excitability profiles for several cortical representations (located closely, or at a distance) by a metric called Earth Mover's Distance (EMD); (2) analysis of the overlaps between any two maps (leading to a creation of a new map). General scheme of the TMSmap possibilities, including an assessment of the standard and novel parameters of TMS motor maps, is presented in Figure 2.

\section{Input Data}

The input data for the software includes structural MRI and the actual results of the stimulation. MRI data is not absolutely necessary for the map reconstruction; it is needed primarily for the visualization of the relationships between the constructed map and the cortical structure. MRI data should be presented in the .nifti format (.img, .hdr files). Both structural MRI data and the stimulation coordinates may be either in the individual coordinates or in the template brain coordinate system, for example, in the MNI coordinate system, in cases of using preprocessed normalized data as in the study of Niskanen et al. (2010).

Data should contain the coordinates of the stimulation points which, in the simplest case, it can be just coil's coordinates on the scalp, as well as the coordinates of the estimated electric field (EF) maximum in the brain. Such coordinates can be obtained using different methodologies: using the line-of-sight approach (like in Raffin et al., 2015), or using EF maximum coordinates precalculated in the neuronavigation system like in Krieg et al. (2017), or using an offline approach with a sophisticated realistic volume conductor modeling of the head [e.g., utilizing a software presented in http://simnibs.de/ like in Bungert et al. (2017)]. For each stimulation point there are associated responses from any number of the recording channels. Current version of TMSmap is primarily designed for motor mapping data. In this case the channels should contain the information about the MEP characteristics such as peak-to-peak amplitude, latency, duration, 


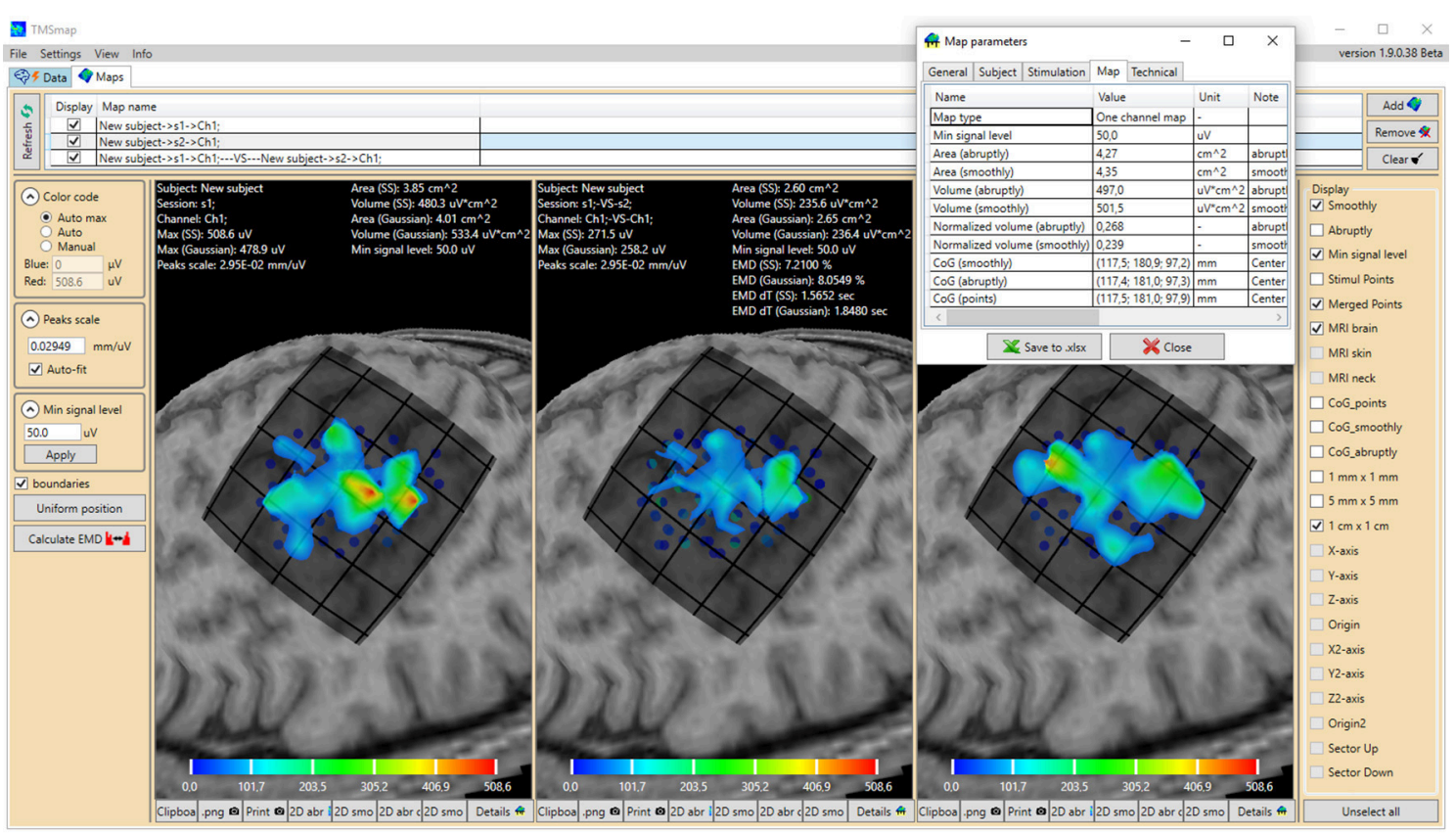

FIGURE 1 | General view of the software interface. Upper panel represents two main tabs of the program: "Data" (preprocessing) and "Maps" (3D map construction and comparison). The tab "Maps" is chosen, cortical representation maps for two muscles (left and right maps) and their overlap (in the middle) are represented. Left panel contains the parameters of the map construction and has a button for EMD calculation. Right panel represents the features which can be vizualized. For each map the low panel represents the possibility to generate pictures and tables of the results. A table with the main information about the map is shown for one of the maps. Color bars are representing the amplitudes of the MEPs in microvolts.

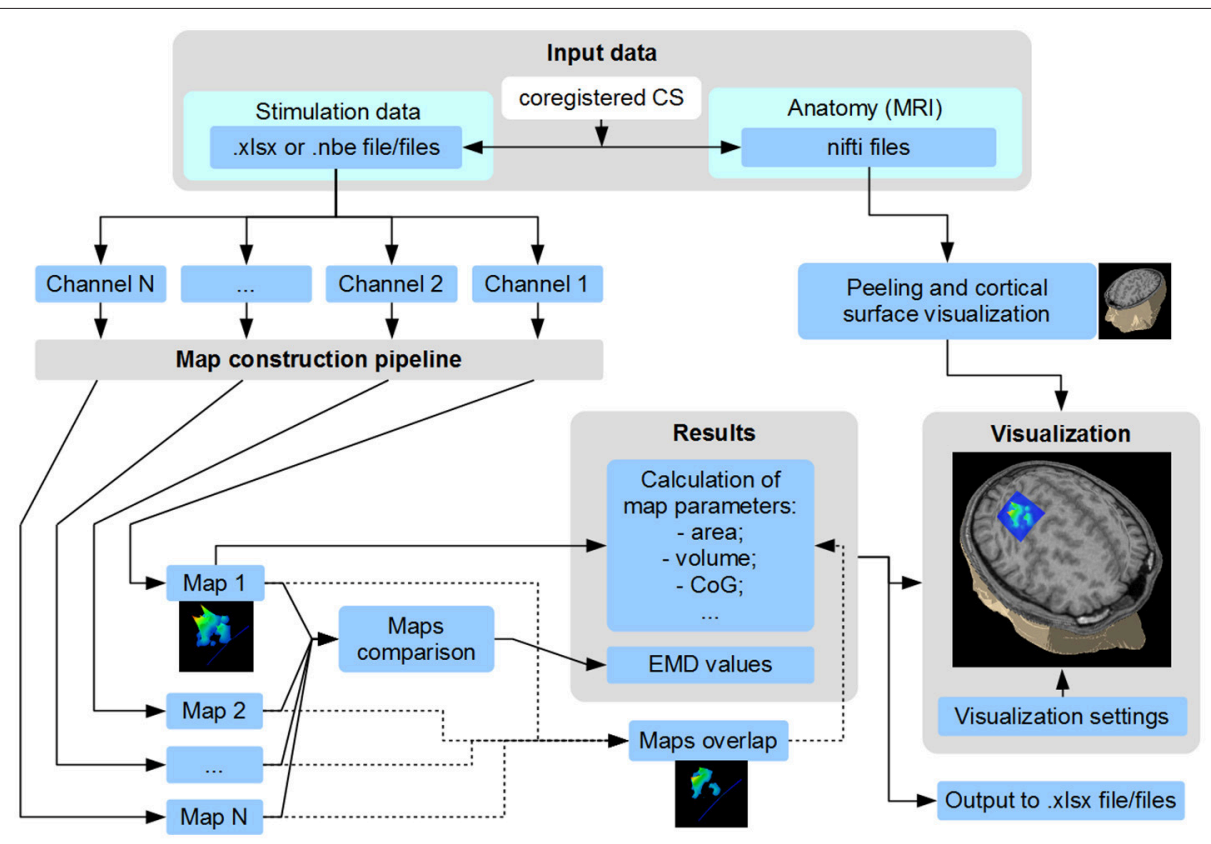

FIGURE 2 | Scheme of TMSmap possibilities. CS, coordinate system; CoG, center of gravity; EMD, earth mover's distance.

number of peaks etc. However, other types of the parameters, for example "Yes", "No" e.g., when inducing phosphenes or error types-can also be used as a response value. Stimulation data can be presented either in .xlsx file or in a text file of .nbe format in case of using Nexstim navigation system. It should be mentioned that TMSmap can be used for the purposes of 
viewing and editing of the stimulation data files (including .nbe files).

A detailed step-by-step procedure of the map construction is provided in the tutorials available at the website.

\section{TMSmap Functions}

\section{Brain Anatomy Visualization}

The definition of the skin surface is based on the data about the tissue density across voxels. The peeling is performed at the minimum distance sufficient for the visualization of all the stimulation points. Then the cortical landscape is visualized based on the tissue density differences between the cortex and the cerebro-spinal fluid.

\section{Construction and Visualization of the Map Surface}

For the construction of the representation surface, TMSmap uses two different approaches. In the first it is assumed that the response function is changing relatively smoothly among the stimulation points (Figure $\mathbf{3 A}$ ). In this case for the surface construction TMSmap uses an approach allowing to create a maximally smooth surface going through all the pointsapproximation based on smoothing (ABOS) method (Dressler, 2009). Another approach for the map surface reconstruction, realized in the TMSmap, is based on the assumption that the response function may change rather abruptly from one point to another. In this case, the constructed surface would be built as a combination of the kernel surfaces defined by the exponential function created from each stimulation point; this approach would be further referred to as a Kernel approach (Figure 3B). Based on the physical principles of TMS it is not possible to stimulate one point without the stimulation of the adjacent ones (Ilmoniemi and Kicić, 2010). Thus, the approach based on the smoothly changing function is preferable for TMS mapping data analysis-this being in agreement with the data presented in the literature (Julkunen, 2014). A Kernel approach is still included in the software since we anticipate in the future a usage of highresolution TMS with the next generation of the stimulators providing more focal stimulation (Koponen et al., 2017). In addition, TMSmap may be also utilized for motor mapping during invasive stimulation of the cortex (for neurosurgical purposes) where the spatial resolution of the stimulation is much higher.

A general schema of the map surface construction consists of the following steps:

1. Obtain coordinates of all the stimulation points with the corresponding response values (e.g., MEP peak-to-peak amplitude). Optionally, a maximum of the induced EF (if available) can be taken into the account for weighting the response values.

2. All the stimulation points should be used for fitting the closest spherical surface which is found using the least squares method (Figure 4a). All stimulation points are projected to this sphere (Figure $4 \mathbf{b}$ ). For maps' comparison all the stimulation points should be fitted to the same spherical surface. Consequently, this part

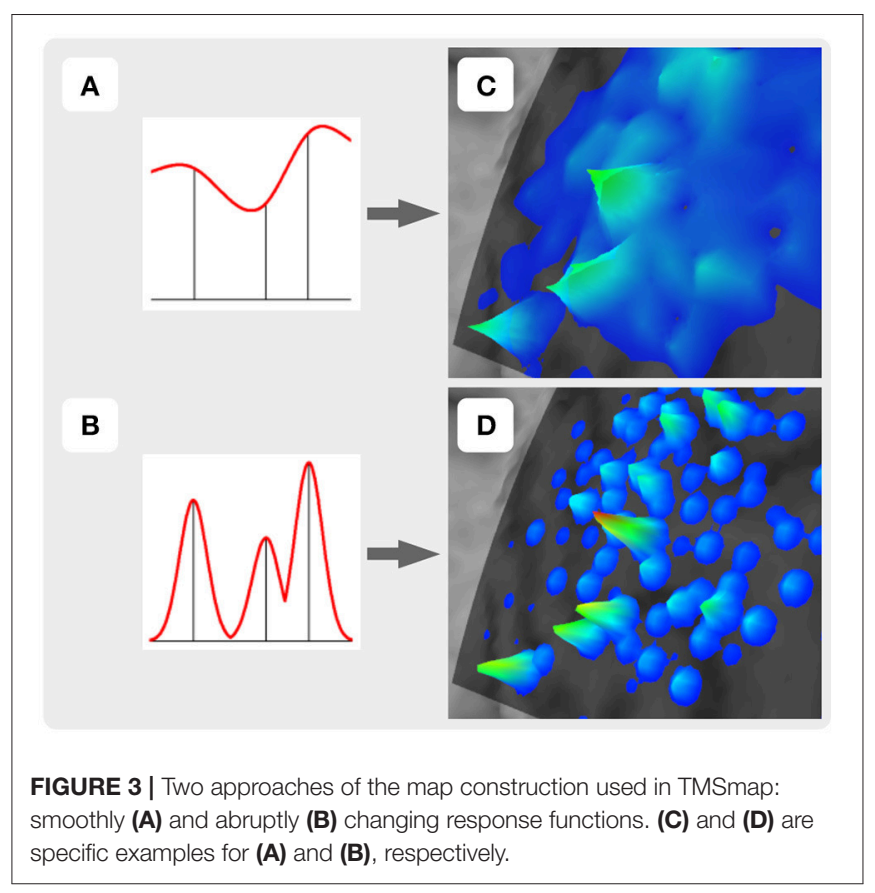

of the sphere surface is defined as shown in Figure $4 \mathbf{b}$ for the further analysis. This part of the sphere surface would be further referred to as a patch of interest (POI).

3. Spatial filtering (merging) is applied to the points (optionally). Therefore, new merged points with the averaged locations and averaged/maximal response values are created (Figure $4 \mathbf{b}$ ). Using this option it is possible to utilize only merged points with sufficient number of the repetitions for map construction.

4. Quasi-regular grid inside the POI is constructed (Figure 4c). 5. In case of abruptly changing function assumption (Figure 4e) Kernel approach is used for map construction. A height of the surface in any arbitrary point inside the POI is defined according to the formula:

$$
\mathrm{h}\left(\mathrm{A}_{\alpha, \beta}\right)=\max _{\mathrm{i}=1 \ldots \mathrm{N}}\left(\mathrm{h}_{\mathrm{i}} \cdot \mathrm{a}^{\left(\frac{\mathrm{r}^{2}\left(\mathrm{~A}_{\alpha, \beta}, \mathrm{A}_{\mathrm{i}}\right)}{\mathrm{b}^{2}}\right)}\right),
$$

where

$\mathrm{A}_{\alpha, \beta}$ - point with the angle coordinates $(\alpha, \beta)$;

$\mathrm{N}$ - a number of the merged projected stimulation points;

$\mathrm{h}_{\mathrm{i}}$ - averaged response value in the merged point $A_{i}$;

a - the portion of the whole kernel volume located outside the radius $b$. Hereby, $b$ is the navigation accuracy in $(1-a)$ portion of all the cases. For example, the default TMSmap settings for $\mathrm{a}-0.05$ and for $\mathrm{b}-2 \mathrm{~mm}$. It means that only $5 \%$ of the whole kernel volume would be more than $2 \mathrm{~mm}$ further 

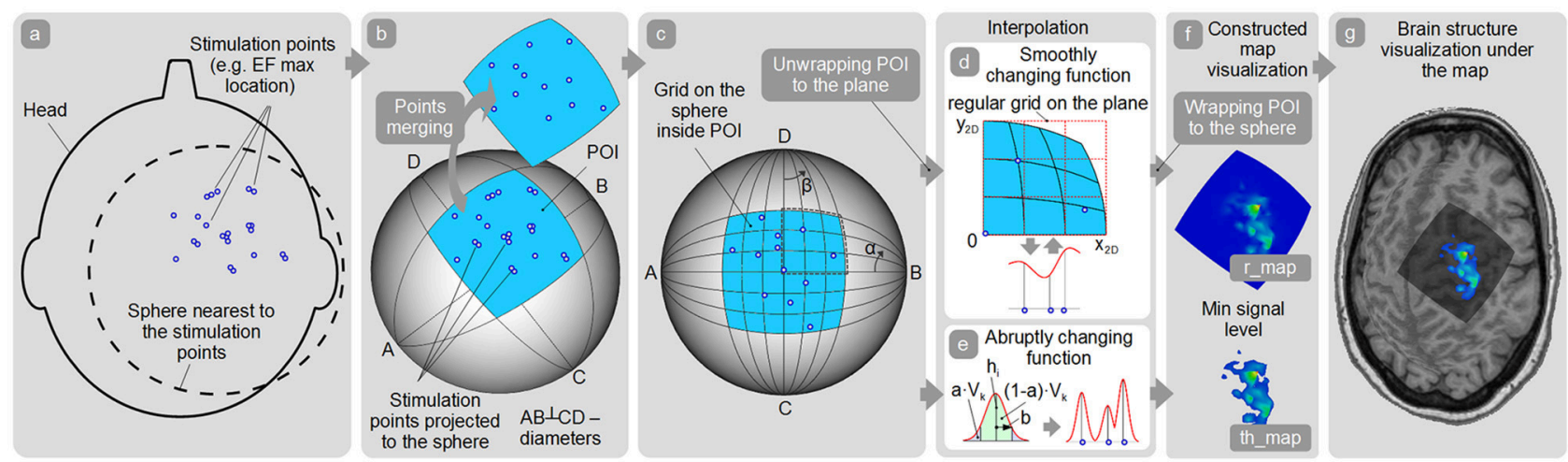

FIGURE 4 | Map construction pipeline. (a) Finding the sphere nearest to the stimulation points; (b) Patch of interest (POI) creation around the projected stimulation points and points spatial merging; (c) Quasi-regular grid creation inside the POI; (d,e) Interpolation among the points in case of smoothly and abruptly changing function approaches; (f) r_map and th_map visualization; (g) Visualization of the representation on the cortex.

from the stimulation point, indicating $95 \%$ probability for the real stimulation point to be at this given area;

$r\left(A_{\alpha, \beta}, A_{i}\right)$ - geodesic distance between points $A_{\alpha, \beta}$ and $\mathrm{A}_{\mathrm{i}}$;

6. In case of using a smoothly changing function approach the found POI with the merged projected points is unwrapped to the plane (Figure $4 \mathbf{d}$ ). Arbitrary POI point $\mathrm{A}_{\alpha, \beta}$ will have plane coordinates:

$$
\mathrm{x}_{2 \mathrm{D}}(\alpha, \beta)=\mathrm{k} \cdot \cos \theta, \mathrm{y}_{2 \mathrm{D}}=\mathrm{k} \cdot \sin \theta
$$

where

$$
\begin{aligned}
& \mathrm{k}=\mathrm{R} \cdot \arccos \left(\frac{\cos \alpha \cdot \cos \beta}{\sqrt{1-\sin ^{2} \alpha \cdot \sin ^{2} \beta}}\right) ; \\
& \theta=\left\{\begin{array}{c}
\arctan \left(\frac{\tan \alpha}{\tan \beta}\right)+\pi \cdot \mathrm{H}(-\beta), \beta \neq 0 \\
\operatorname{sign}(\alpha) \cdot \pi / 2, \beta=0
\end{array}\right.
\end{aligned}
$$

$\mathrm{R}$ - Radius of the sphere where the stimulation points are projected;

$\mathrm{H}$ - Heaviside step function.

A regular grid is created around the unwrapped POI on the plane. Grid's element size is not a user-customized parameter. Additionally, user can define a maximal radius of the stimulation point influence which is a distance where an approximated map surface is reaching zero level (default TMSmap settings: $5 \mathrm{~mm}$ ).

At this stage map construction on the plane is performed using ABOS-approach (Dressler, 2009). After that a regular grid with the POI is wrapped back to the sphere:

$$
\alpha=\arctan \left(\frac{\mathrm{x}_{3 \mathrm{D}}}{\mathrm{z}_{3 \mathrm{D}}}\right), \beta=\arctan \left(\frac{\mathrm{y}_{3 \mathrm{D}}}{\mathrm{z}_{3 \mathrm{D}}}\right),
$$

where

$$
\begin{aligned}
\mathrm{x}_{3 \mathrm{D}} & =\frac{\mathrm{R} \cdot \sin \frac{\mathrm{s}}{\mathrm{R}}}{\sqrt{1+\left(\frac{\mathrm{y}_{2 \mathrm{D}}}{x_{2 D}}\right)^{2}}}, \mathrm{y}_{3 \mathrm{D}}=\mathrm{x}_{3 \mathrm{D}} \cdot \frac{\mathrm{y}_{2 \mathrm{D}}}{\mathrm{x}_{2 \mathrm{D}}}, \mathrm{z}_{3 \mathrm{D}}=\mathrm{R} \cdot \cos \frac{\mathrm{s}}{\mathrm{R}} \\
\mathrm{s} & =\sqrt{\mathrm{x}_{2 \mathrm{D}}{ }^{2}+\mathrm{y}_{2 \mathrm{D}^{2}}}
\end{aligned}
$$

7. The constructed map is defined as a raw map - r_map for the further analysis (Figure 4f).

8. An additional color coding of the created response map is possible using a continuous color scale from blue ( $\min$ response value level) to red ( $\max$ response value level).

9. A minimum signal level surface is created based on the user-defined threshold (e.g., MEP amplitude equal to $50 \mu \mathrm{V}$ for standard area calculation or a percentage of the largest MEP within the map for highest excitability regions identification etc.). This surface is concentric to the sphere where stimulation points were projected. Only the part of the response surface which is above this minimum signal level surface will be taken for the further analysis, it will be defined as a thresholded map_th_map (Figure 4f).

10. Optionally, brain structure is visualized under the constructed map (Figure 4g).

The default values of the map construction parameters proposed in TMSmap are explained in the manual. In order to compare results of the different studies, all the parameters should be ideally the same. Of course, this does not prevent the user from exploring different parameters' values.

\section{Construction of the Maps' Overlap}

It is possible to choose any of the two constructed maps (e.g., channel 1 and channel 2) to create their overlap map (Figure 2). The overlap of the other maps can be calculated iteratively for any 
two pairs of the maps. The overlap maps have the same set of the properties as the channel's maps.

\section{Area and Volume Calculation}

Map area is defined as an area of the projection of the th_map to POI. Map volume is calculated as a volume under this th_map surface.

\section{CoG Calculation}

CoGs locations for raw data and for the constructed 3D-maps are calculated according to the following equations:

$$
\begin{aligned}
\mathrm{x}_{\mathrm{CoG}}^{\mathrm{raw}} & =\frac{\sum_{\mathrm{i}=1}^{\mathrm{N}}\left(\mathrm{h}_{\mathrm{i}} \cdot \mathrm{x}_{\mathrm{i}}\right)}{\sum_{\mathrm{i}=1}^{\mathrm{N}} \mathrm{h}_{\mathrm{i}}} ; \\
\mathrm{x}_{\mathrm{CoG}}^{\text {th_map }} & =\frac{\sum_{\mathrm{i}=1}^{\mathrm{N}_{\mathrm{E}}}\left(\mathrm{h}_{\mathrm{E} \_\mathrm{i}} \cdot \mathrm{s}_{\mathrm{i}} \cdot \mathrm{x}_{\mathrm{E} \_\mathrm{i}}\right)}{\sum_{\mathrm{i}=1}^{\mathrm{N}_{\mathrm{E}}}\left(\mathrm{h}_{\mathrm{E} \_\mathrm{i}} \cdot \mathrm{s}_{\mathrm{i}}\right)},
\end{aligned}
$$

where in case of raw data

$\mathrm{N}$ - number of the merged points;

$h_{i}$ and $x_{i}$ - averaged response value and $x$ coordinate of the merged point, respectively;

and in case of th_map

$\mathrm{N}_{\mathrm{E}}$ - number of the grid's elements belonging to the th_map;

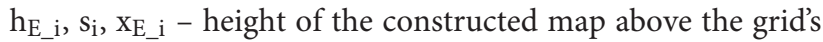
element, area and x coordinate of the center of the grid's element, consequently.

\section{Creation of the Color-Coded 2D Maps}

There is an option to create separate files of color-coded pictures for each map (Figure 5). These files are saved in .png format for the further analysis.

\section{Maps 3D Profiles Comparison-EMD}

Map surface could be characterized by its individual 3D profile. TMSmap allows numerical comparison of the profiles of both th_map and the distributions of the raw (not interpolated) response values using the so-called Wasserstein metric or EMD (Rubner et al., 1998, 2000). EMD is a minimum amount of work

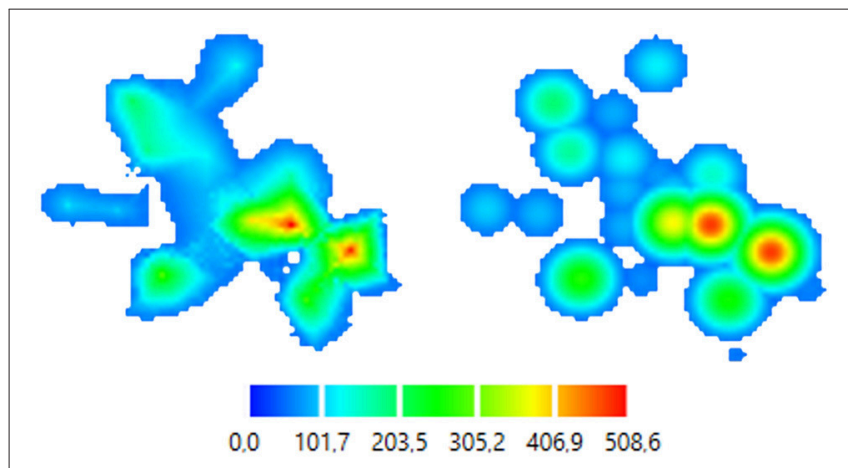

FIGURE 5 | Color-coded 2D map representations. Left-smoothly changing function approach (ABOS based map construction), Right - the same data, abruptly changing function approach (Kernel based map construction). Color bar is representing the amplitudes of the MEPs in microvolts. needed to shift one distribution to another. The notion of "work" in case of maps comparison would be a geodesic distance between the stimulation points. EMD may be useful to numerically differentiate maps located nearby to each other. These maps might be similar in terms of the standard parameters such as areas and volumes but still varying due to the complexity of their excitability profiles. Implementation of the EMD metric in TMSmap is based on the modification of the open-source $\mathrm{C}++$ code (http://ai.stanford.edu/ rubner/emd/default.htm\#LOG). In TMSmap the two maps should be normalized by volume before calculating EMD between them. Then EMD is calculated and represented as a relative value - a percentage of the EMD between two extreme maps each consisting of the only one peak maximally separated from each other in the limits of the individual size of the "active" area. For each subject individually we define "active area" in the following manner: we include any stimulated point where at least one muscle in at least one session had a response with the amplitude higher than a chosen threshold (e.g., MEP amplitude $>50 \mu \mathrm{V}$ ). A maximum distance between the points in such active area is taken as an individual constant for the EMD normalization.

\section{Results Output}

All calculated parameters are represented in the results tables (Figure 1) and can be copied or saved as an .xlsx file.

\section{Visualization}

TMSmap allows offline visualization of the multiple features of the TMS mapping results such as:

1. Stimulation points;

2. POI;

3. Stimulation points projected to the POI;

4. Spatially merged points with color-coded averaged response values;

5. Minimum signal level surface - a user-customized threshold surface;

6. r_map and th_map built based on one of the two possible approaches (ABOS or Kernel);

7. 3 types of CoGs based on the above-mentioned approaches;

8. Overlaps of any of the two maps;

9. 2D color-coded figures of any map, including overlap maps; 10. Different size grids on the POI.

All mapping features can be visualized in relation to an individual brain MRI. All visualization parameters can be represented independently or in parallel.

\section{EXAMPLES}

In this section, we will provide examples of TMS motor mapping using datasets from two healthy subjects and one chronic ischemic stroke patient with a favorable hand motor recovery. We previously presented an example of the earlier version of the software for TMS motor mapping in healthy and stroke subjects in the $\mathrm{PhD}$ thesis of one of the authors (Nazarova, 2015). All TMS investigations were carried out in accordance with the safety TMS guidelines (Rossi et al., 2009), subjects 
participating in the TMS mapping procedure gave a written informed consent in accordance with the Declaration of Helsinki. All subjects were screened for contraindications to TMS (Rossi et al., 2009) before the consenting process. Experiments were approved by the local Ethics Committees of the Research Center of Neurology and Higher School of Economics (Moscow) and TMS motor mapping was performed in the Research Center of Neurology, Moscow. A Nexstim eXimia stimulator with nTMS-compatible electromyography (EMG) device, navigation software, and a figure-of-eight coil (Focal Bipulse, Nexstim Plc, Helsinki, Finland) was used for the stimulation. Structural T1 MRIs required for the navigation were acquired with $1.5 \mathrm{~T}$ MR-scanner Siemens Magnetom Avanto (T1 weighted; $1 \mathrm{~mm}$ thickness; sagittal orientation; acquisition matrix $256 \times 256$ ). As a first step a "hotspot" of the cortical representation of the abductor pollicis brevis (APB) muscle was found in the primary motor cortex. The resting motor threshold (RMT) for the given "hotspot" was determined as a minimal stimulator output producing contralateral APB MEPs with a minimal amplitude being $50 \mu \mathrm{V}$ in a resting muscle, in 5 out of 10 given stimuli (Rossini et al., 1994). The intensity of the stimulation during the mapping procedure was always kept at $110 \%$ of the RMT for APB. Minimal time lag between the stimuli was $3 \mathrm{~s}$. Further details for the mapping procedure are described below.

We used data from two healthy right-handed male volunteers (30 and 28 years old). For these volunteers two TMS motor mapping sessions separated by 7 days were performed (Day 1 and Day 2). The sessions consisted of nTMS mapping of the cortical representation of the three right hand muscles: $\mathrm{APB}$, abductor digiti minimi (ADM) and extensor digitorum communis (EDC). Each of the two mapping sessions included 5 sub-sessions each including 55 or 53 TMS pulses for subject 1 and 2, respectively. The stimulation nodes were pre-set using a virtual MRI-based grid, so that each of the point was stimulated in a pseudo-random order five times. Day 2 points were stimulated in the exactly same order as on the Day 1. An error of the navigation for each cortical spot was kept below $2 \mathrm{~mm}$. RMT for APB was 35 and $31 \%$ for the volunteer 1 and 2, respectively.

For the third example we used the data from a chronic stroke patient (male, 55 years old, 20 months after the incident) with the cortical-subcortical ischemic lesion in the left hemisphere and favorable upper limb motor recovery ( 55 by Fugl-Meyer upper extremity scale). The RMTs for APB was $51 \%$ for the unaffected hemisphere (UH) and 75\% for the affected hemisphere (AH). A total amount of the points was 120 for the $\mathrm{UH}$ and 125 for the $\mathrm{AH}$, each spot was stimulated only once.

The MEP amplitudes were calculated online in the eXimia software. During the initial preprocessing EMG data were visually inspected. Only a few MEPs had to be rejected due to concurrent muscle contraction. We used precalculated EF maximum location coordinates provided by the navigation software. Further analysis was performed in the TMSmap. The following possibilities of the TMSmap are shown: a visualization of the excitability profiles of the TMS muscle cortical representations and their overlaps; a possibility to visualize the stimulation points and the merged points based on the chosen criteria for spatial filtering; calculation of the areas and

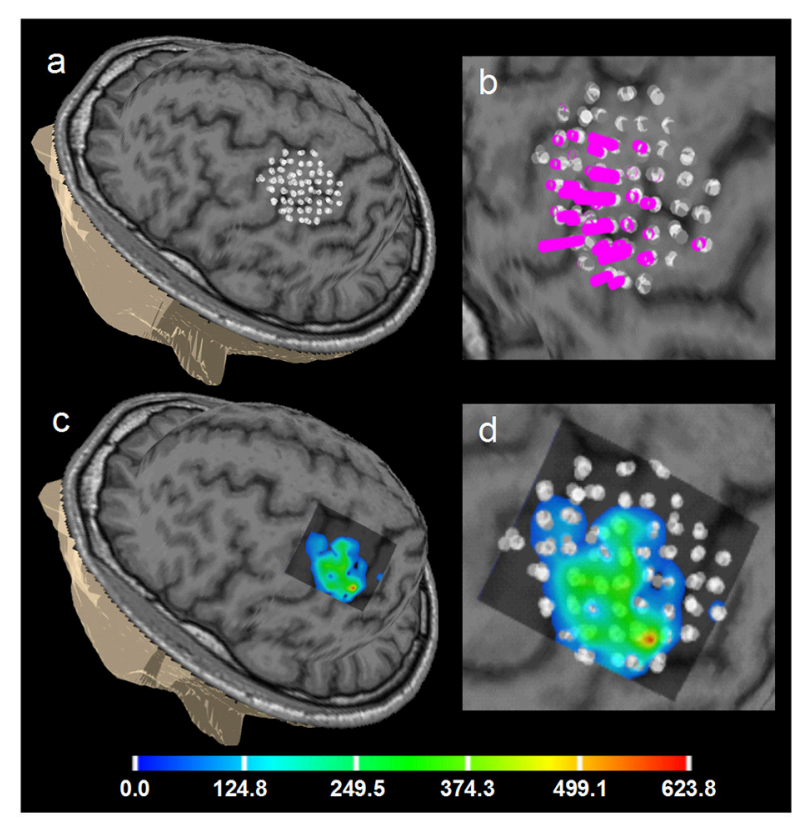

FIGURE 6 | Healthy volunteer 1. ABOS based visualization of the APB cortical representation, a summation of 5 sessions of the first day, not merged stimulation points are visualized to show the accuracy of the stimulation of the same point in 5 sub-sessions. (a) points visualized on the brain surface; (b) its zoomed view with the perpendiculars from the points, height of the perpendiculars reflects the MEPs amplitudes. (c) ABOS based APB map reconstruction on the brain surface and (d) its zoomed view. Color scale representing amplitudes of the MEPs in microvolts is shown.

volumes of the motor maps and their overlaps; comparison of the $3 \mathrm{D}$ excitability profiles of the different sessions and different muscles using EMD metric.

Figure 6 shows an example of the right APB cortical surface map for the first healthy subject. Stimulation points without applying spatial filtering (merging) are shown to demonstrate an accuracy of the repeated stimulation of the same point in 5 subsessions. In Figure 7 an example of the test-retest mapping data from the healthy volunteer 2 is presented. Here spatial filtering (merging) of the stimulation points was used. The similarity of the $3 \mathrm{D}$ profiles of the different maps measured using EMD metric is shown.

Figure 8 is an example of multi-muscle TMS mapping in the ischemic stroke patient. A greater difference between APB and EDC representations in the $\mathrm{AH}$ compared to the $\mathrm{UH}$ could be observed, it is manifested as a shrinkage of the APB and an extension of the EDC cortical representation. EMD values reflecting the differences in the topographies are also shown.

\section{DISCUSSION}

We have developed and introduced TMSmap—novel standalone software with a graphical interface for the quantitative analysis of the nTMS mapping results. To the best of our knowledge until now, there was no such versatile software for the analysis of TMS 


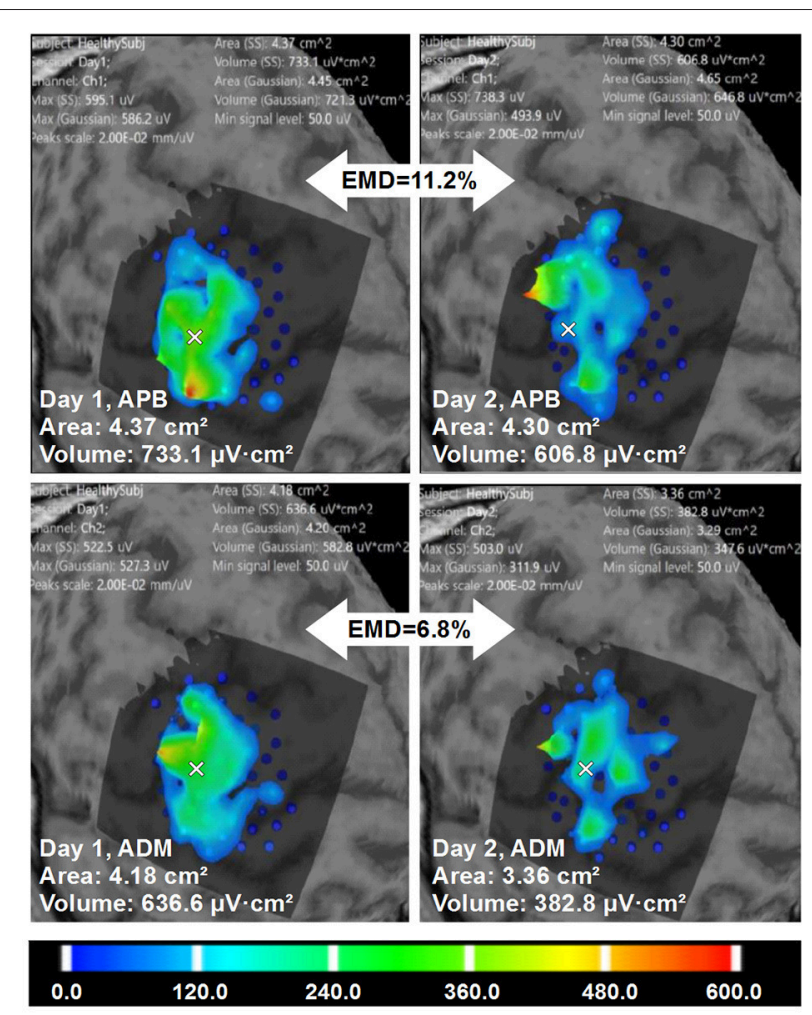

FIGURE 7 | Healthy volunteer 2. The visualization of the ABOS based surface reconstruction of APB and ADM maps in both days (already based on five sessions for each day, merged points with more than two repetitions in each). CoGs are shown with a white cross. It is worth noting that APB maps in Day 1 and Day 2 are more similar in terms of areas and volumes. However, the excitability profiles reliability is higher for ADM cortical representation. Color scale reflecting amplitudes of the MEPs in microvolts is shown.

mapping results. Previous approaches were primarily based on custom-made scripts for Matlab (Niskanen et al., 2010; Kraus and Gharabaghi, 2016; van de Ruit and Grey, 2016) which makes the comparison and generalization of the results between groups a challenge. In this article we described the functionality of TMSmap and methods used for the construction and quantitative assessment of the cortical surface maps. Consequently, we illustrated its performance for the analysis of TMS motor maps in two healthy subjects and in one stroke patient. TMSmap was used for the assessment of similarities of cortical representations of different muscles and of same muscles in different days.

Here we focused primarily on the motor nTMS mapping as one of the most common applications of nTMS mapping (Ruohonen and Karhu, 2010; Lefaucheur and Picht, 2016). It is important to mention that until now, even for the estimation of a muscle's cortical representation area there is no standard approach (Julkunen, 2014) and its reliability in testretest studies is rather questionable (Kraus and Gharabaghi, 2016). Therefore, the use of a standardized tool covering diverse steps of TMS mapping pipeline would facilitate comparison among studies, which is needed for multi-center studies and meta-analysis relating to TMS motor mapping in different conditions.

Moreover, despite being the most widely used metric, TMS cortical representation of a muscle is a rather challenging biological concept, especially when considering well-known principles of the motor cortex organization such as divergence and convergence (Schieber, 2001; Capaday et al., 2013; Nazarova and Blagovechtchenski, 2015). Apparently, measuring just an area of a given muscle cortical representation doesn't take into account these principles. TMS mapping results should not be interpreted as if the neuronal activation occurs at only one small brain point but rather that the induced motor responses are obtained for given coordinates of the coil or for the coordinates of the strongest values of the induced EF. TMS mapping still might be used for more comprehensive studies of motor cortex organization. This could be achieved for example by assessing relationships among the cortical representation of different muscles and by analyzing the regions where TMS produces simultaneous responses in several muscles. Such an approach takes into account both divergence and convergence phenomena. Indeed, in animal studies it was shown that around $50 \%$ of the cortico-motoneuronal cells recorded with microelectrodes facilitate at least one proximal and at least one distal muscle (McKiernan et al., 1998). Moreover, it was demonstrated that depending on the limb position, microstimulation at the same point may evoke activity in different muscles (Graziano, 2006). Interestingly, a similar effect was demonstrated during one spot TMS, where a concept of "selectivity ratio" was introduced, defined as the amplitude of the MEP elicited from the muscle depending on whether it acts as an agonist or antagonist in the following movement (Gerachshenko et al., 2008; Uehara et al., 2015). However, there are still only a few studies on TMS mapping dedicated specifically to the muscle representation overlap phenomenon. It is clear that such overlap is a prominent phenomenon (Wassermann et al., 1993; Devanne et al., 2006). Moreover, it was shown that there was, in fact, a difference in the extent of such overlap in dominant and non-dominant hemispheres (Melgari et al., 2008). In addition, it was demonstrated that the extent of this overlap can be changed in some pathological conditions like dystonia or chronic pain (Schabrun et al., 2009, 2015). One of the prominent hypotheses states that the amount of the overlap among different populations of cortical motor cells may represent a neural substrate for creating muscle synergies (Capaday et al., 2013). This, in turn, may be extrapolated to the TMS representations' overlaps. Moreover, there is already some evidence for this idea (Schabrun et al., 2009, 2015; Massé-Alarie et al., 2017). Thus, the continuation of the TMS investigation of cortical representations' overlaps appears to be a promising approach. Our software includes calculations of all parameters of the overlaps among different cortical representations, thus, allowing an estimation of corresponding plastic changes in longitudinal studies.

Another important parameter proposed in TMSmap is based on a convergence principle of motor cortex organization. It relates to the excitability profile of muscle cortical representation. The convergence principle indicates that different loci along the pre-central gyrus contain separate representations of the 


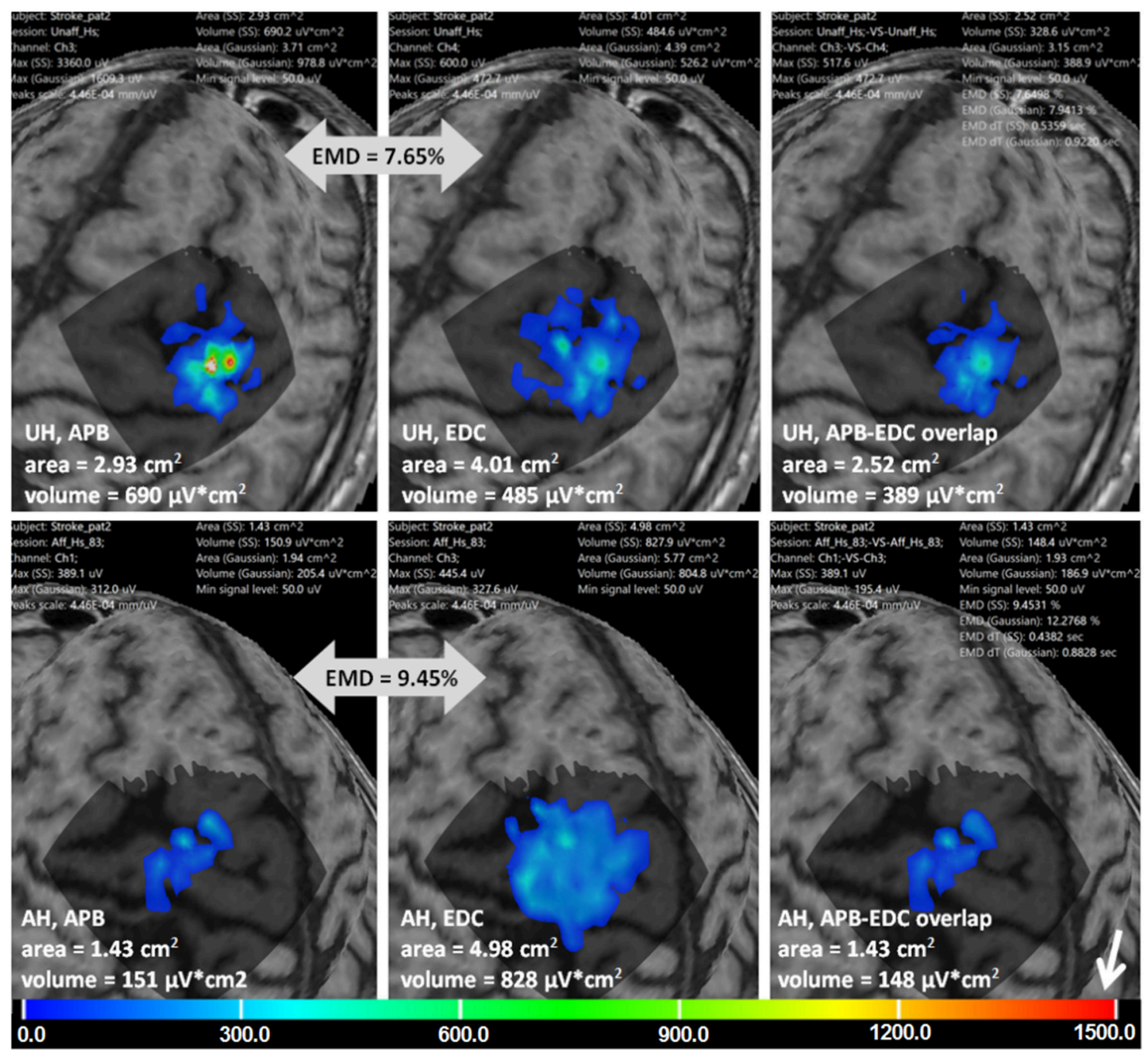

FIGURE 8 | Stroke patient. APB and EDC cortical representations and their overlaps using ABOS based surface reconstruction are created for both hemispheres. Maps' areas and volumes are visualized. Relative EMD values among APB and EDC representations are shown (big gray arrows). Color scale representing amplitudes of the MEPs in microvolts is shown with a small white arrow.

same muscle (Schieber, 2001). In animal studies it was clearly shown that the pyramidal neurons relating to a single digit muscle are widely distributed in the motor cortex and may even be found in the regions which are traditionally known to contain shoulder representation (Rathelot and Strick, 2006). In many TMS studies it was shown that the representation of a single muscle is widely distributed over the motor cortex (Wassermann et al., 1992; Lotze et al., 2003; Melgari et al., 2008). It was reported that muscle representation can have discrete islands of relatively stable responses (Littmann et al., 2013) and parts of the representation with higher excitability so-called "peaks of excitability" may be physiologically relevant for the understanding of motor outputs (Massé-Alarie et al., 2017). However, still in many TMS studies the variability among MEPs amplitudes in the cortical representation is rather ignored (Sollmann et al., 2013; Ruit et al., 2014). Recently it has been reported that in the case of TMS motor mapping consisting of only seven cortical spots along the central sulcus, it was possible to trace an excitability profile of the responses from several hand muscles characterized by partial somatotopy both during rest and during the isometric contraction of one of the muscles (Raffin et al., 2015). However, such line mapping may not be sufficient for mapping purposes in clinical studies considering the fact that even hotspots of the hand muscles can be located not on the "hand knob" (Ahdab et al., 2016). Therefore, a distributed excitability profile analysis including a wide grid of the stimulated points might be more advantageous. The question of the biological meaning of any parameter is tightly connected with its stability in normal conditions. In TMS map we proposed the way to analyze similarity of such excitability profiles using EMD, a metric which allows evaluation of the dissimilarity between two multi-dimensional distributions (Rubner et al., 
1998, 2000; Haufe et al., 2008). Thus, the next step would be to investigate "the phenomenon of the excitability profile reliability" in a test-retest study-exemplary results are presented here for one of the healthy subjects.

TMSmap may be widely used for purposes of quantitative analysis and offline visualization of TMS motor mapping results, including longitudinal studies. Standardizing the procedure of map construction and their parameters would lead to an easier comparison of results, thus, being particularly relevant for studying reorganization in the course of a disease, rehabilitation or training. Indeed, until now in order to investigate TMS motor maps, the changes were analyzed either by visual assessment (Mäkelä et al., 2013) or using parameters such as areas of the representations, CoGs location and a few other characteristics alternating from study to study (Littmann et al., 2013; Ruit et al., 2014; Sankarasubramanian et al., 2015; Kraus and Gharabaghi, 2016). Multi-parametric assessment of the TMS motor maps including standard and novel parameters accessible in TMSmap may reveal more subtle changes in cortical organization. The simplest example of TMSmap usage is a classical TMS motor mapping with computation of MEPs amplitudes elicited by single pulse TMS applied to different cortical points. At the same time, any other parameter, detected with TMS-EMG approach, such as the latency or duration of MEP, the extent of paired-pulse TMS phenomena, intensity necessary to induce a silent period etc. may also be easily utilized for motor map reconstruction in TMSmap. Such comprehensive analysis of motor maps would be especially valuable in fields of neurorehabilitation and neuroenhancement where even small modifications of the map might be important for estimating the effects of intervention. Indeed, TMS mapping has already been shown to have a potential diagnostic/therapeutic value in pathological conditions such as stroke (Lüdemann-Podubecká and Nowak, 2016), amyotrophic lateral sclerosis (Chervyakov et al., 2015), dystonia (Thickbroom et al., 2003; Quartarone, 2013), multiple sclerosis (Thickbroom et al., 2005), pain therapy (Nurmikko et al., 2016) etc, as well as in healthy subjects under experimental conditions such as immobilization, training or neuromodulation (Ngomo et al., 2012; Boudreau et al., 2013) or in special populations such as musicians (Elbert and Rockstroh, 2004), sportsmen (Hänggi et al., 2015) or surgery trainees. TMSmap may supposedly increase this potential. Finally, TMSmap capabilities are not restricted to the purposes of TMS motor mapping. Considering the fact that the necessary input data includes anatomical coordinates and any type of numerical/rank response, the software can be used for 3D map construction of other responses relating to behavioral performance, EEG, fMRI etc.

\section{LIMITATIONS AND OUTLOOK}

A cortical surface map construction is at the core of TMSmap, thus leading to the standardization of the mapping results. Evidently, other factors such as accuracy of the navigation system, as well as stimulation procedure itself are important sources of the TMS results' variability. However, just excluding the variability relating to map construction and parameters' calculation, should be beneficial for the assessment of TMS results. One limitation of TMSmap is that currently it has been tested with only three available TMS navigation systems: two commercial ones: Nexstim, Localite and one open-sourceInVesalius neuronavigation (https://github.com/invesalius/ invesalius $3 /$ tree/master/navigation/mtc_files). The latter two provide only information about the coil position in contrast to the Nexstim system, where in addition one has precalculated EF maximum coordinates based on the spherical model (Krieg, 2017). Further updates to the program will include compatibility with other navigation systems and the implementation of the existing types of EF distribution modeling (e.g., like Pitkänen et al., 2017). Currently, EF maximum coordinates (calculated with another software presented in http://simnibs.de/) can be used. In the future, we plan to provide ranges for the expected normal fluctuation of standard and novel parameters of the motor TMS maps in healthy subjects utilizing present and future reproducibility studies.

Link to the website http://tmsmap.ru/ - software for quantitative analysis of TMS mapping results.

\section{AUTHOR CONTRIBUTIONS}

PN developed the software, developed some of the methods used in the software, tested the software, wrote the manuscript, and agreed to be accountable for all aspects of the work. MN generated the idea, supervised the whole project, performed TMS mapping experiments, tested the software, wrote the manuscript and agreed to be accountable for all aspects of the work. VVN participated in the development of the software, discussion and writing of the manuscript, he agreed to be accountable for all aspects of the work. PN and MN share the first authorship and have contributed equally to the study.

\section{ACKNOWLEDGMENTS}

Authors are deeply grateful to Dr. A. Ossadchi and Dr. A. Zhdanov for discussions. Authors are also grateful to Dr. V.H. Souza and MS R. Matsuda for the data from the navigation system InVesalius provided for testing. Example data of two healthy participants and one stroke subject were obtained in the Research Center of Neurology, Moscow while MN was working there in the years 2012-2016. We acknowledge Dr. Y. Rubner for the open-source code for EMD calculation the modification of which we used. Authors are also grateful to MS I. Gusarovas and to the students D. Pozdeeva, E. Ivanina, and K. Kozlova for software testing. The article was prepared within the framework of the Basic Research Program at the National Research University Higher School of Economics (HSE) and was partly supported within the framework of a subsidy by the Russian Academic Excellence Project 5-100. MN and PN were partly funded by RFBR grant no. 16-04-01883. MN was partly supported by Skolkovo personal grant Umnik. 


\section{REFERENCES}

Ahdab, R., Ayache, S. S., Brugières, P., Farhat, W. H., and Lefaucheur, J. P. (2016). The hand motor hotspot is not always located in the hand knob: a neuronavigated transcranial magnetic stimulation study. Brain Topogr. 29, 590-597. doi: 10.1007/s10548-016-0486-2

Boudreau, S. A., Lontis, E. R., Caltenco, H., Svensson, P., Sessle, B. J., Andreasen Struijk, L. N., et al. (2013). Features of cortical neuroplasticity associated with multidirectional novel motor skill training: a TMS mapping study. Exp. Brain Res. 225, 513-526. doi: 10.1007/s00221-012-3391-2

Bungert, A., Antunes, A., Espenhahn, S., and Thielscher, A. (2017). Where does TMS Stimulate the Motor Cortex? Combining electrophysiological measurements and realistic field estimates to reveal the affected cortex position. Cereb. Cortex 27, 5083-5094. doi: 10.1093/cercor/bhw292

Capaday, C., Ethier, C., Van Vreeswijk, C., and Darling, W. G. (2013). On the functional organization and operational principles of the motor cortex. Front. Neural Circuits 7:66. doi: 10.3389/fncir.2013.00066

Chervyakov, A. V., Bakulin, I. S., Savitskaya, N. G., Arkhipov, I. V., Gavrilov, A. V., Zakharova, M. N., et al. (2015). Navigated transcranial magnetic stimulation in amyotrophic lateral sclerosis. Muscle Nerve 51, 125-131. doi: $10.1002 /$ mus. 24345

Devanne, H., Cassim, F., Ethier, C., Brizzi, L., Thevenon, A., and Capaday, C. (2006). The comparable size and overlapping nature of upper limb distal and proximal muscle representations in the human motor cortex. Eur. J. Neurosci. 23, 2467-2476. doi: 10.1111/j.1460-9568.2006.04760.x

Dressler, M. M. (2009). Art of Surface Interpolation. Kuni Tát. Available at: http:// m.dressler.sweb.cz/AOSIM.pdf (Accessed December 9, 2016).

Elbert, T., and Rockstroh, B. (2004). Reorganization of human cerebral cortex: the range of changes following use and injury. Neuroscientist 10, 129-141. doi: $10.1177 / 1073858403262111$

Gerachshenko, T., Rymer, W. Z., and Stinear, J. W. (2008). Abnormal corticomotor excitability assessed in biceps brachii preceding pronator contraction poststroke. Clin. Neurophysiol. 119, 683-692. doi: 10.1016/j.clinph.2007.11.004

Graziano, M. (2006). The organization of behavioral repertoire in motor cortex. Annu. Rev. Neurosci. 29, 105-134. doi: 10.1146/annurev.neuro.29.051605.112924

Groppa, S., Oliviero, A., Eisen, A., Quartarone, A., Cohen, L. G., Mall, V., et al. (2012). A practical guide to diagnostic transcranial magnetic stimulation: report of an IFCN committee. Clin. Neurophysiol. 123, 858-882. doi: 10.1016/j.clinph.2012.01.010

Hänggi, J., Langer, N., Lutz, K., Birrer, K., Mérillat, S., and Jäncke, L. (2015). Structural brain correlates associated with professional handball playing. PLoS ONE 10:e0124222. doi: 10.1371/journal.pone.0124222

Haufe, S., Nikulin, V. V., Ziehe, A., Müller, K. R., and Nolte, G. (2008). Combining sparsity and rotational invariance in EEG/MEG source reconstruction. Neuroimage 42, 726-738. doi: 10.1016/j.neuroimage.2008. 04.246

Ilmoniemi, R. J., and Kicić, D. (2010). Methodology for combined TMS and EEG. Brain Topogr. 22, 233-248. doi: 10.1007/s10548-0090123-4

Julkunen, P. (2014). Methods for estimating cortical motor representation size and location in navigated transcranial magnetic stimulation. J. Neurosci. Methods 232, 125-133. doi: 10.1016/j.jneumeth.2014.05.020

Koponen, L. M., Nieminen, J. O., and Ilmoniemi, R. J. (2017). Multi-locus TMS: electronic control of the stimulation location with large thin overlapping coils. Brain Stimul. 10, 441. doi: 10.1016/j.brs.2017.01.314

Kraus, D., and Gharabaghi, A. (2016). Neuromuscular plasticity: disentangling stable and variable motor maps in the human sensorimotor cortex. Neural Plast. 2016, 1-13. doi: 10.1155/2016/7365609

Krieg, S. M. (ed.). (2017). Navigated transcranial magnetic stimulation in Neurosurgery (Springer International Publishing), 299.

Krieg, S. M., Lioumis, P., Mäkelä, J. P., Wilenius, J., Karhu, J., Hannula, H., et al. (2017). Protocol for motor and language mapping by navigated TMS in patients and healthy volunteers; workshop report. Acta Neurochir. 159, 1187-1195. doi: 10.1007/s00701-017-3187-z

Lefaucheur, J. P., and Picht, T. (2016). The value of preoperative functional cortical mapping using navigated TMS. Neurophysiol. Clin. Neurophysiol. 46, 125-133. doi: 10.1016/j.neucli.2016.05.001
Littmann, A. E., McHenry, C. L., and Shields, R. K. (2013). Variability of motor cortical excitability using a novel mapping procedure. J. Neurosci. Methods 214, 137-143. doi: 10.1016/j.jneumeth.2013.01.013

Lotze, M., Kaethner, R. J., Erb, M., Cohen, L. G., Grodd, W., and Topka, H. (2003). Comparison of representational maps using functional magnetic resonance imaging and transcranial magnetic stimulation. Clin. Neurophysiol. 114, 306-312. doi: 10.1016/s1388-2457(02)00380-2

Lüdemann-Podubecká, J., and Nowak, D. A., (2016). Mapping cortical hand motor representation using TMS: A method to assess brain plasticity and a surrogate marker for recovery of function after stroke? Neurosci. Biobehav. Rev. 69, 239-251. doi: 10.1016/j.neubiorev.2016.07.006

Mäkelä, J. P., Vitikainen, A. M., Lioumis, P., Paetau, R., Ahtola, E., Kuusela, L., et al. (2013). Functional plasticity of the motor cortical structures demonstrated by navigated TMS in two patients with epilepsy. Brain Stimul. 6, 286-291. doi: 10.1016/j.brs.2012.04.012

Massé-Alarie, H., Bergin, M. J. G., Schneider, C., Schabrun, S., and Hodges, P. W. (2017). "Discrete peaks" of excitability and map overlap reveal task-specific organization of primary motor cortex for control of human forearm muscles. Hum. Brain Mapp. 38, 6118-6132. doi: 10.1002/hbm.23816

McKiernan, B. J., Marcario, J. K., Karrer, J. H., and Cheney, P. D. (1998). Corticomotoneuronal postspike effects in shoulder, elbow, wrist, digit, and intrinsic hand muscles during a reach and prehension task. J. Neurophysiol. 80, 1961-1980. doi: 10.1152/jn.1998.80.4.1961

Melgari, J. M., Pasqualetti, P., Pauri, F., and Rossini, P. M. (2008). Muscles in "Concert": Study of primary motor cortex upper limb functional topography. PLoS ONE 3, 1-11. doi: 10.1371/journal.pone.0003069

Nazarova, M. А. (2015). Мультимодальная Оценка Реорганизации Двигательной Системы Руки После Полушарного Инсульта: МРТТМС Исследование. Диссертация к.м.н. Available online at: http://www. neurology.ru/sites/default/files/assets/documents/2015/12/disser-nazarovama_0.pdf?download=1 (Accessed November 8, 2017).

Nazarova, M., and Blagovechtchenski, E. (2015). Modern brain mapping - what do we map nowadays? Front. Psychiatry 6:89. doi: 10.3389/fpsyt.2015.00089

Ngomo, S., Leonard, G., and Mercier, C. (2012). Influence of the amount of use on hand motor cortex representation: Effects of immobilization and motor training. Neuroscience 220, 208-214. doi: 10.1016/j.neuroscience.2012.06.018

Niskanen, E., Julkunen, P., Säisänen, L., Vanninen, R., Karjalainen, P., and Könönen, M. (2010). Group-level variations in motor representation areas of thenar and anterior tibial muscles: navigated transcranial magnetic stimulation study. Hum. Brain Mapp. 31, 1272-1280. doi: 10.1002/hbm.20942

Nurmikko, T., MacIver, K., Bresnahan, R., Hird, E., Nelson, A., and Sacco, P. (2016). Motor cortex reorganization and repetitive transcranial magnetic stimulation for pain-a methodological study. Neuromodulation Technol. Neural Interface 19, 669-678. doi: 10.1111/ner.12444

Pitkänen, M., Kallioniemi, E., Julkunen, P., Nazarova, M., Nieminen, J. O., and Ilmoniemi, R. J. (2017). Minimum-norm estimation of motor representations in Navigated TMS mappings. Brain Topogr. 30, 711-722. doi: 10.1007/s10548-017-0577-8

Quartarone, A. (2013). Transcranial magnetic stimulation in dystonia. Handb. Clin. Neurol. 116, 543-553. doi: 10.1016/B978-0-444-53497-2.0 0043-7

Raffin, E., Pellegrino, G., Di Lazzaro, V., Thielscher, A., and Siebner, H. R. (2015). Bringing transcranial mapping into shape: sulcus-aligned mapping captures motor somatotopy in human primary motor hand area. Neuroimage 120, 164-175. doi: 10.1016/j.neuroimage.2015.07.024

Rathelot, J. A., and Strick, P. L. (2006). Muscle representation in the macaque motor cortex: an anatomical perspective. Proc. Natl. Acad. Sci. U.S.A. 103, 8257-8262. doi: 10.1073/pnas.0602933103

Rossi, S., Hallett, M., Rossini, P. M., and Pascual-Leone, A. (2009). Safety, ethical considerations, and application guidelines for the use of transcranial magnetic stimulation in clinical practice and research. Clin. Neurophysiol. 120, 2008-2039. doi: 10.1016/j.clinph.2009.08.016

Rossini, P. M., Barker, A. T., Berardelli, A., Caramia, M. D., Caruso, G., Cracco, R. Q., et al. (1994). Non-invasive electrical and magnetic stimulation of the brain, spinal cord and roots: basic principles and procedures for routine clinical application. Report of an IFCN committee. Electroencephalogr. Cin. Neurophysiol. 91, 79-92. doi: 10.1016/0013-4694(94)9 0029-9 
Rubner, Y., Tomasi, C., and Guibas, L. J. (1998). “A metric for distributions with applications to image databases," in Sixth International Conference on Computer Vision (Bombay: Narosa Publishing House), 59-66.

Rubner, Y., Tomasi, C., and Guibas, L. J. (2000). The earth mover's distance as a metric for image retrieval. Int. J. Comput. Vis. 40, 99-121. doi: 10.1023/A:1026543900054

Ruit, M., Van De, Perenboom, M. J. L., and Grey, M. J. (2014). TMS brain mapping in less than two minutes. Brain Stimul. 8, 231-239. doi: 10.1016/j.brs.2014.10.020

Ruohonen, J., and Karhu, J. (2010). Navigated transcranial magnetic stimulation. Neurophysiol. Clin. 40, 7-17. doi: 10.1016/j.neucli.2010.01.006

Sankarasubramanian, V., Roelle, S. M., Bonnett, C. E., Janini, D., Varnerin, N. M., Cunningham, D. A., et al. (2015). Reproducibility of transcranial magnetic stimulation metrics in the study of proximal upper limb muscles. J. Electromyogr. Kinesiol. 25, 754-764. doi: 10.1016/j.jelekin.2015.05.006

Schabrun, S. M., Hodges, P. W., Vicenzino, B., Jones, E., and Chipchase, L. S. (2015). Novel adaptations in motor cortical maps: the relation to persistent elbow pain. Med. Sci. Sports Exerc. 47, 681-690. doi: 10.1249/MSS.0000000000000469

Schabrun, S. M., Stinear, C. M., Byblow, W. D., and Ridding, M. C. (2009). Normalizing motor cortex representations in focal hand Dystonia. Cereb. Cortex 19, 1968-1977. doi: 10.1093/cercor/bhn224

Schieber, M. H. (2001). Constraints on somatotopic organization in the primary motor cortex. J. Neurophysiol. 86, 2125-2143. doi: 10.1152/jn.2001.86.5.2125

Sollmann, N., Hauck, T., Obermüller, T., Hapfelmeier, A., Meyer, B., Ringel, F., et al. (2013). Inter- and intraobserver variability in motor mapping of the hotspot for the abductor policis brevis muscle. BMC Neurosci. 14:94. doi: 10.1186/1471-2202-14-94

Thickbroom, G. W., Byrnes, M. L., Archer, S. A., Kermode, A. G., and Mastaglia, F. L. (2005). Corticomotor organisation and motor function in multiple sclerosis. J. Neurol. 252, 765-771. doi: 10.1007/s00415-005-0728-9
Thickbroom, G. W., Byrnes, M. L., Stell, R., and Mastaglia, F. L. (2003). Reversible reorganisation of the motor cortical representation of the hand in cervical dystonia. Mov. Disord. 18, 395-402. doi: 10.1002/mds. 10383

Uehara, K., Coxon, J. P., and Byblow, W. D. (2015). Transcranial direct current stimulation improves ipsilateral selective muscle activation in a frequency dependent manner. PLOS ONE 10:e0122434. doi: 10.1371/journal.pone.01 22434

van de Ruit, M., and Grey, M. J. (2016). The TMS map scales with increased stimulation intensity and muscle activation. Brain Topogr. 29, 56-66. doi: 10.1007/s10548-015-0447-1

Wassermann, E. M., McShane, L. M., Hallett, M., and Cohen, L. G. (1992). Noninvasive mapping of muscle representations in human motor cortex. Electroencephalogr. Clin. Neurophysiol. $85,1-8$.

Wassermann, E. M., Pascual-Leone, A., Valls-Solé, J., Toro, C., Cohen, L. G., and Hallett, M. (1993). Topography of the inhibitory and excitatory responses to transcranial magnetic stimulation in a hand muscle. Electroencephalogr. Clin. Neurophysiol. 89, 424-433.

Conflict of Interest Statement: The authors declare that the research was conducted in the absence of any commercial or financial relationships that could be construed as a potential conflict of interest.

Copyright (๑) 2018 Novikov, Nazarova and Nikulin. This is an open-access article distributed under the terms of the Creative Commons Attribution License (CC BY). The use, distribution or reproduction in other forums is permitted, provided the original author(s) and the copyright owner(s) are credited and that the original publication in this journal is cited, in accordance with accepted academic practice. No use, distribution or reproduction is permitted which does not comply with these terms. 